\title{
Densidade crítica ao crescimento de plantas considerando água disponível e resistência à penetração de um Argissolo Vermelho distrófico arênico
}

\author{
Bulk density critical to the growth of plants considering available water and soil resistance to penetration \\ of a Paleudalf
}

\author{
Cláudia Liane Rodrigues de Lima* ${ }^{\mathrm{I} *}$ José Miguel Reichert $^{\mathrm{I}}$ Dalvan José Reinert ${ }^{\mathrm{I}}$ \\ Luis Eduardo Akiyoshi Sanches Suzuki ${ }^{\text {II }}$ Leandro Dalbianco ${ }^{\text {III }}$
}

\section{- NOTA -}

\section{RESUMO}

O estabelecimento de culturas está associado a condições restritivas impostas pelo tipo e pelo manejo de solo. O objetivo deste estudo foi avaliar o intervalo de densidade crítica de um Argissolo Vermelho distrófico arênico, considerando a disponibilidade de água para as culturas e os valores restritivos de resistência à penetração $(1,5 ; 2,0 ; 2,5$; 3,0 e 3,5MPa). A amostragem de um Argissolo Vermelho distrófico arênico sob plantio direto desde 1989, com estrutura de solo preservada, foi feita nas camadas de 0,00 a 0,10 m e 0,10 a 0,20m. As amostras (oito repetições) foram saturadas em água, equilibradas em diferentes potenciais (-0,001; -0,004; -0,006; -0,033; -0,07 e-0,1MPa) e dois grupos de amostras foram secadas em laboratório, por um período de 7 e 9 dias, perfazendo um total de 128 amostras. Após atingir o equilíbrio, foi avaliada a resistência à penetração e a densidade do solo. Um incremento na resistência à penetração possibilitou maiores intervalos de densidade adequados ao desenvolvimento das plantas, i.e., quando considerada uma resistência à penetração de $2 \mathrm{MPa}$, o intervalo de densidade adequado foi de $1,44 \mathrm{Mg}$ $\mathrm{m}^{-3}$ a $1,76 \mathrm{Mg} \mathrm{m}^{-3}$ e, para uma resistência de $3 \mathrm{MPa}$, o intervalo foi de 1,53 a $1,88 \mathrm{Mg} \mathrm{m}^{-3}$. Valores superiores e inferiores ao intervalo de densidade obtidos são críticos ao desenvolvimento de culturas, considerando a resistência à penetração e a disponibilidade de água.

Palavras-chave: umidade do solo, densidade do solo, resistência à penetração.

\section{ABSTRACT}

Crop establishment is linked to restrictive conditions imposed by soil type and soil management. This study aimed at evaluating the interval of bulk density critical for a Paleudalf, considering water availability to the crops and restrictive values to the root resistance to penetration $(1.5 ; 2.0 ; 2.5 ; 3.0 \mathrm{e}$ $3.5 \mathrm{MPa}$ ). Soil samples, from a Paleudalf under no-tillage since 1989, with preserved structure were collected in the layers of 0.00 to $0.10 \mathrm{~m}$ and 0.10 to $0.20 \mathrm{~m}$ depth. The samples (eight replicates) were saturated in water and balanced different potentials (- 0.001; -0.004; -0.006; -0.033; -0.07 and $0.1 \mathrm{MPa}$ ), plus 2 groups of soil samples were dried in the laboratory for a period of 7 and 9 days, being a total of 128 samples used in this study. After reaching the balance, the soil resistance to penetration and bulk density were evaluated. An increase in the resistance to penetration allows for large intervals of bulk density no restrictive to the plant development. For instance, when considered a resistance to penetration of $2 \mathrm{MPa}$, the adequate interval of bulk density was $1.44 \mathrm{Mg} \mathrm{m}^{-3}$ to $1.76 \mathrm{Mg}$ $\mathrm{m}^{-3}$, and for a resistance to penetration of $3 \mathrm{MPa}$ the interval was 1.53 to $1.88 \mathrm{Mg} \mathrm{m}^{-3}$. Bulk density smaller or larger than interval obtained are critical to the development of plants, considering soil resistance to penetration and water availability.

Key words: soil moisture, bulk density, resistance to penetration.

O potencial produtivo agrícola é determinado por fatores relacionados ao solo e à espécie envolvida. Funcionalmente, uma estrutura de solo ideal é aquela que permite um espaço poroso suficiente e contínuo para o movimento de água e gases e uma resistência do solo à penetração não impeditiva ao crescimento de raízes e da parte aérea da planta (KOPI\& DOUGLAS, 1991).

A definição de uma estrutura de solo ideal é dificultada pela variabilidade temporal e espacial de indicadores físicos e pela interdependência de vários

IDepartamento de Solos, Centro de Ciências Rurais (CCR), Universidade Federal de Santa Maria (UFSM), 97105-900, Santa Maria, RS, Brasil. E-mail: clrlima@yahoo.com.br.*Autor para correspondência.

IIDepartamento de Ciências Florestais, CCR, UFSM, Santa Maria, RS, Brasil. E-mail: suzuki@mail.ufsm.br.

${ }^{\text {IIIC } C u r s o ~ d e ~ A g r o n o m i a, ~ U F S M, ~ S a n t a ~ M a r i a, ~ R S, ~ B r a s i l . ~ E-m a i l: ~ e s p e t o u f s m @ y a h o o . c o m . b r . ~}$ 
atributos. Outro aspecto que tem gerado muitas contestações está relacionado ao conceito de disponibilidade de água às plantas. A disponibilidade de água às plantas é definida pelo intervalo compreendido entre o limite superior e o inferior de água disponível, caracterizado pela umidade do solo equivalente à capacidade de campo e ao ponto de murcha permanente (CASSEL \& NIELSEN, 1986).

Diferentes valores de parâmetros físicos restritivos ao crescimento de plantas têm sido indicados na literatura científica. TAYLOR et al. (1966) consideram valores de resistência à penetração superiores a $2 \mathrm{MPa}$ impeditivos ao crescimento e ao funcionamento do sistema radicular. No entanto, existe carência de informações que indiquem valores de densidade restritiva ao desenvolvimento radicular das plantas.

Há necessidade de ampliar as pesquisas nessa área de conhecimento que possibilitem prever o grau de degradação da estrutura e orientar práticas de manejo do solo e da água. Considerando que valores de densidade restritivos ao crescimento e estabelecimento de culturas dependem do tipo, das condições e do manejo do solo, o objetivo deste estudo foi avaliar o intervalo de densidade crítica para as plantas de um Argissolo Vermelho distrófico arênico, considerando a disponibilidade de água para as culturas e a resistência do solo à penetração radicular.

Este estudo foi realizado no ano de 2005, em uma área sob plantio direto desde 1989 (delineamento inteiramente casualizado), pertencente ao Departamento de Solos da Universidade Federal de Santa Maria, UFSM, RS (29 45’ S; 53 42” W; 95m de altitude). O solo foi um Argissolo Vermelho distrófico arênico (EMBRAPA, 1999) de textura superficial francoarenosa (81 $\mathrm{g} \mathrm{kg}^{-1}$ de argila, $291 \mathrm{~g} \mathrm{~kg}^{-1}$ de silte e $628 \mathrm{~g} \mathrm{~kg}^{-1}$ de areia). O clima da região é caracterizado como Cfa (subtropical úmido sem estiagens), de acordo com a classificação de Köppen, sendo a temperatura média do mês mais quente superior a $22^{\circ} \mathrm{C}$ e a do mês mais frio entre $-3^{\circ} \mathrm{C}$ e $18^{\circ} \mathrm{C}$.

A amostragem com estrutura de solo preservada foi realizada com cilindros de Uhland $(0,076 \mathrm{~m}$ de altura e 0,076m de diâmetro interno), nas camadas de 0,00 a $0,10 \mathrm{~m}$ e 0,10 a 0,20m. Com o objetivo de obter um gradiente de umidade, as amostras foram saturadas e equilibradas a diferentes potenciais matriciais ( $\Psi)$ em mesa de tensão e em panelas de pressão utilizando placas de Richards (KLUTE, 1986). Foram utilizados os $\Psi$ de: - 0,001; -0,004; -0,006; -0,033; -0,07 e -0,1MPa, e mais dois grupos de amostras de solo secadas ao ar no laboratório por períodos de 7 e 9 dias. Para cada camada de coleta e potencial matricial, foram utilizadas oito repetições, perfazendo um total de 128 amostras. Após atingir o equilíbrio, foi medida em cada amostra a resistência do solo a partir de um penetrômetro de taxa constante de penetração, dotado de ponta cônica com ângulo de penetração de $30^{\circ}$ e $12,83 \mathrm{~mm}$ de diâmetro da base e com armazenamento eletrônico de dados (COLLARES et al., 2004).

Os resultados de resistência à penetração foram ajustados em relação à umidade volumétrica $(\theta$, $\mathrm{m}^{3} \mathrm{~m}^{-3}$ ) e à densidade do solo (Ds, $\mathrm{Mg} \mathrm{m}^{-3}$ ), utilizando o modelo não linear proposto por BUSSCHER (1990): $R P=a \theta^{b} D s$, em que: RP é a resistência do solo à penetração (MPa), e $a, b$ e $c$ são parâmetros empíricos do modelo obtidos mediante ajustes não lineares. A partir desse modelo, quantificou-se a faixa de densidade do solo crítica (Dsc) utilizando-se valores de resistência à penetração de 1,5; 2,0; 2,5; 3,0 e 3,5MPa e umidades na capacidade de campo e no ponto de murcha permanente.

A umidade na capacidade de campo foi avaliada a campo, saturando-se uma área de aproximadamente $1 \mathrm{~m}^{2}$. Durante um período de 17 dias, utilizando-se um trado holandês, foi quantificada a umidade gravimétrica nas camadas de 0,00 a $0,10 \mathrm{~m}$ e 0,10 a $0,20 \mathrm{~m}$. Considerou-se a umidade na capacidade de campo quando essa manteve-se quase constante ao longo do tempo. O ponto de murcha fisiológico foi quantificado por COLLARES (2005), em solos coletados na camada de $0,00-0,20 \mathrm{~m}$, sendo destorroados, homogeneizados e acondicionados em vasos, avaliando-se as espécies vegetais feijão, soja e girassol, obtendo-se um valor médio de umidade gravimétrica no ponto de murcha de $0,051 \mathrm{~kg}^{1} \mathrm{~kg}^{-1}$.

A amplitude de variação da densidade do solo $\left(1,37 \mathrm{Mg} \mathrm{m}^{-3}<\mathrm{Ds}<1,73 \mathrm{Mg} \mathrm{m}^{-3}\right)$ e da resistência à penetração $(0,20 \mathrm{MPa}<\mathrm{RP}<4,34 \mathrm{MPa})$ estiveram relacionadas a diferentes condições de umidade, profundidade de amostragem e variabilidade deste solo.

Verificou-se que os parâmetros de ajuste ( $a=0,004 ; b=-1,580$ e $c=6,389)$ e o modelo utilizado foram significativos $\left(\mathrm{P}<0,0001, \mathrm{R}^{2}=0,83\right)$. A partir dos parâmetros do modelo, estabeleceu-se a relação entre RP, $\theta$ e Ds (Figura 1a). Uma relação positiva entre a Ds e à RP também foi demonstrada por GERARD et al. (1982), PRADO et al. (2002) e LAMPURLANÉS \& CANTERO-MARTÍNEZ (2003), em diferentes tipos de solos e sistemas de preparo. Da mesma forma, uma relação negativa entre RP e $\theta$ (Figura 1a) foi observada por BUSSCHER et al. (1997) e VAZ \& HOPMANS (2001). O aumento da densidade pode condicionar um novo arranjamento e acomodamento das partículas do solo favorecendo um incremento do número de contato entre elas, aumentando a resistência à penetração e diminuindo a porosidade do solo (SILVA et al., 2002). 


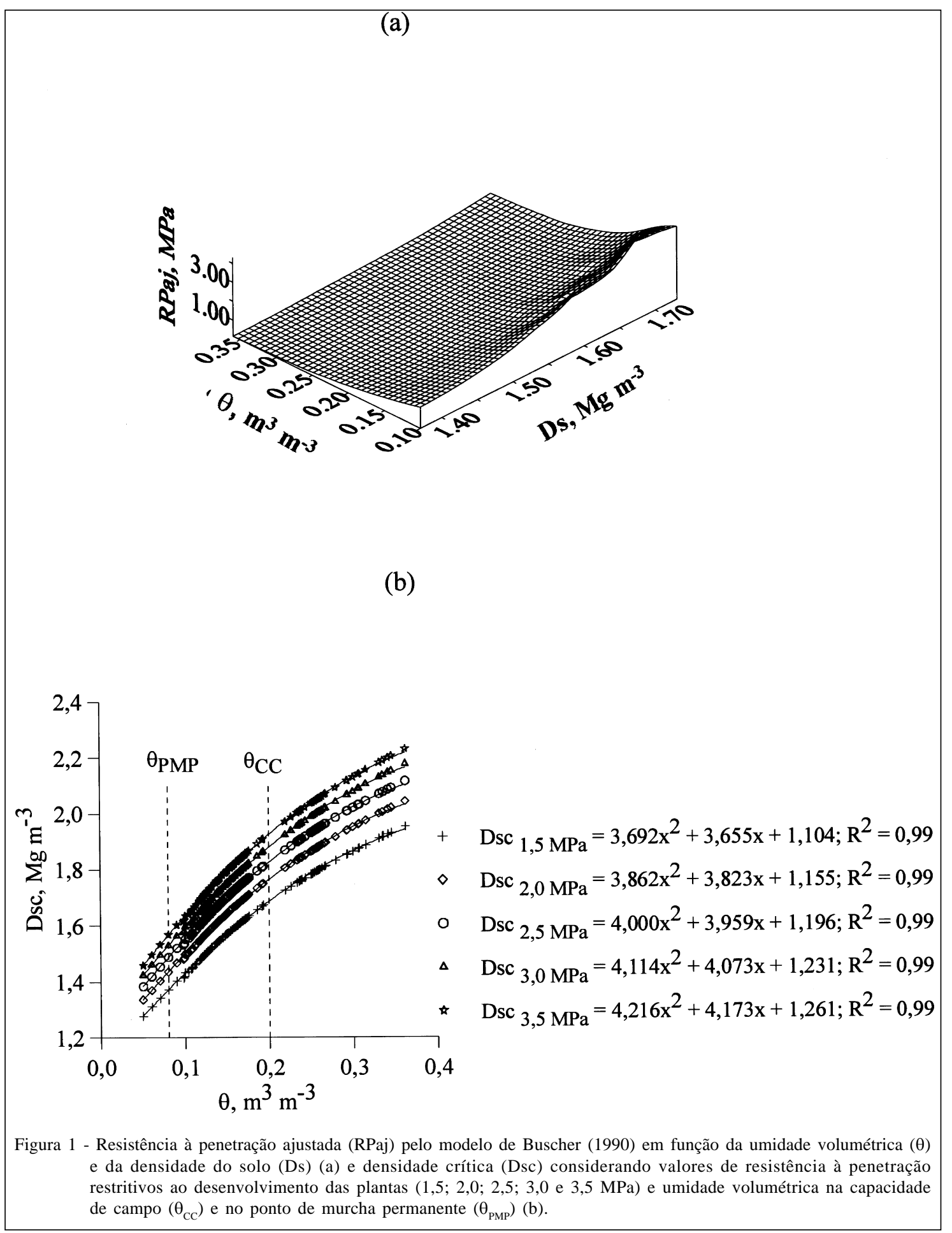

A Figura $1 \mathrm{~b}$ apresenta a faixa de densidade crítica ao crescimento de plantas, considerando a RP e a disponibilidade de água para as culturas (intervalo de umidade entre a capacidade de campo e o ponto de murcha permanente). Verificou-se que, com o aumento de umidade, valores maiores de densidade do solo são possíveis sem que seja superada a resistência à penetração crítica ao desenvolvimento radicular.

Adotando-se uma $\mathrm{RP}=1,5 \mathrm{MPa}$, valores de densidade inferiores a 1,37 e superiores a $1,69 \mathrm{Mg} \mathrm{m}^{-3}$ podem ser restritivos ao crescimento radicular. Quando considerada uma $\mathrm{RP}=2 \mathrm{MPa}$, a densidade crítica (Dsc) 
na $\theta_{\mathrm{CC}}$ foi de $1,76 \mathrm{Mg} \mathrm{m}^{-3} \mathrm{e}$ na umidade no ponto de murcha permanente $\left(\theta_{\mathrm{PMP}}\right)$ foi de $1,44 \mathrm{Mg} \mathrm{m}^{-3}$. TAYLOR et al. (1966) verificaram limitações ao crescimento radicular na resistência à penetração de $2 \mathrm{MPa}$. Porém, esses mesmos autores verificaram crescimento nulo com $\mathrm{RP}=2,5 \mathrm{MPa}$. Considerando uma resistência à penetração de 2,5MPa, valores de densidade inferiores a 1,49 e superiores a $1,83 \mathrm{Mg} \mathrm{m}^{-3}$ podem ser limitantes ao desenvolvimento das plantas. Por outro lado, RPs de 3 a 3,5MPa indicavam, respectivamente, intervalos de densidade adequados ao crescimento de culturas de 1,53 a 1,88 $\mathrm{Mg} \mathrm{m}^{-3}$ e de 1,57 a 1,93Mg m ${ }^{-3}$. Resistência à penetração de $3,5 \mathrm{MPa}$ também é indicada como restritiva por outros autores (MEROTTO JR. \& MUNDSTOCK, 1999).

Informações importantes relacionadas ao manejo do solo e de irrigação de culturas podem ser obtidas a partir da avaliação de indicadores facilmente mensuráveis como a densidade e a umidade do solo. Considerando-se um valor médio de densidade de $1,58 \mathrm{Mg} \mathrm{m}^{-3}$ encontrado neste estudo, ela não representa um fator crítico para o desenvolvimento das plantas (Figura 1b). Observou-se que valores superiores de RP permitiram maiores intervalos de densidade adequados ao desenvolvimento das plantas e que a relação entre a Dsc e a umidade representa grande relevância no monitoramento e manejo adequado da estrutura e da umidade do solo, para um satisfatório crescimento e desenvolvimento das plantas.

\section{REFERÊNCIAS}

BUSSCHER, W.J. Adjustment of flat-tipped penetrometer resistance data to a commom water content. Transactions of the American Society of Agricultural Engineers, v.3, p.519-524, 1990.

BUSSCHER, W.J. et al. Correction of cone index for soil water content differences in a coastal plain soil. Soil and Tillage Research, v.43, p.205-217, 1997.

CASSEL, D.K.; NIELSEN, D.R. Field capacity and available water capacity. In: KLUTE, A. (Ed). Methods of soil analysis: physical and mineralogical methods. 2.ed. Madison: American Society of Agronomy. 1986. p.902-926.

COLLARES, G.L. et al. Desenvolvimento e funcionalidade de um penetrômetro de cone com taxa constante de penetração no solo. In: REUNIÃO BRASILEIRA DE MANEJO E CONSERVAÇÃO DO SOLO E DA ÁGUA, 15., 2004, Santa Maria. Anais... Santa Maria: SBCS, 2004. (CD-ROM)

COLLARES, G.L. Compactação em Latossolos e Argissolo e relação com parâmetros de solo e de plantas. 2005. 106f. Tese (Doutorado em Ciência do Solo) - Curso de Pósgraduação em Solos, Universidade Federal de Santa Maria.

EMPRESA BRASILEIRA DE PESQUISA AGROPECUÁRIA. Centro Nacional de Pesquisa de Solos. Sistema Brasileiro de Classificação de Solos. Brasília: Embrapa produção de informação; Rio de Janeiro: Embrapa Solos, 1999. 412p.

GERARD, C.J. et al. Physical factors influencing soil strength and root growth. Agronomy Journal, v.74, p.875-879, 1982.

KOPI, A.J.; DOUGLAS, J.T. A rapid inexpensive and quantitative procedure for assessing soil structure with respect to cropping. Soil Use and Management, v.7, p.52-56, 1991.

KLUTE, A. Water retention: laboratory methods. In: KLUTE, A. (Ed). Methods of soil analysis: physical and mineralogical methods. 2.ed. Madison: American Society of Agronomy, 1986. Cap.26, p.635-660.

LAMPURLANÉS, J.; CANTERO-MARTÍNEZ, C. Soil bulk density and penetration resistance under different tillage and crop management systems and their relationship with barley root growth. Agronomy Journal, v.95, p.526-536, 2003.

MEROTTO JR., A.; MUNDSTOCK, C.M. Wheat root growth as affected by soil strength. Revista Brasileira de Ciência do Solo, v.23, p.197-202, 1999.

PRADO, R. de M. et al. Sistemas de preparo e resistência à penetração e densidade de um Latossolo Vermelho eutrófico em cultivo intensivo e pousio. Pesquisa Agropecuária Brasileira, v.7, p.1795-1801, 2002.

SILVA, V.R. et al. Fatores controladores da compressibilidade de um Argissolo Vermelho Amarelo distrófico arênico e de um Latossolo Vermelho distrófico típico. I - Estado inicial de compactação. Revista Brasileira de Ciência do Solo, v.26, p.1-8, 2002.

TAYLOR, H.M. et al. Soil strength-root penetration relations for medium-to coarse-textured soil materials. Soil Science, v.102, p.18-22, 1966.

VAZ, C.M.P.; HOPMANS, J.W. Simultaneous measurement of soil penetration resistance and water content with a combined penetrometer - TDR moisture probe. Soil Science Society of America Journal, v.65, p.4-12, 2001. 